\title{
DIABETES AND HEART FAILURE
}

\author{
Trinath Kumar Mishra1, Surakishore Mishra²
}

1 Professor, Department of Cardiology, MKCS Medical College.

${ }^{2}$ Associate Professor, Department of Cardiology, MKCS Medical College.

ABSTRACT

\section{BACKGROUND}

Diabetes ranks among most common chronic disease in the world. In the year 2015, 415 million people were suffering from diabetes and the number is expected to swell to 642 millions in the year 2042. Hypertension, ischaemic heart disease and valvular heart disease increase the risk of Heart Failure (HF) in diabetes. In absence of these also, diabetics are more vulnerable for HF. This has led to the concept of diabetic cardiomyopathy. Diabetic cardiomyopathy can present as HF with reduced ejection fraction or preserved ejection fraction. Treatment is with diuretics, ACE inhibitors, ARBS and Beta blockers.

\section{KEYWORDS}

Diabetes, Heart Failure, Cardiomyopathy.

HOW TO CITE THIS ARTICLE: Mishra TK, Mishra S. Diabetes and heart failure. J. Evolution Med. Dent. Sci. 2017;6(34):2852-2855, DOI: $10.14260 /$ Jemds/2017/613

\section{BACKGROUND}

The global population of diabetes is projected to increase from 382 million in 2013 to 592 million in 2035. Premature Cardiovascular Disease (CVD) is the leading cause of morbidity and mortality in diabetics. The risk of developing CVD in diabetics is twice that of non-diabetics and $80 \%$ of the mortality in diabetics is due to CVD/Coronary Heart Disease is the most important cause of mortality as well as heart failure in diabetics, but the incidence of heart failure in diabetics remains high even after adjustment not only for CAD and hypertension but also race, level of education, cigarette smoking, alcohol consumption, obesity, ACE inhibitors, beta-blockers, diabetes type and duration and interim myocardial infarction. Historically, the concept of a specific diabetic cardiomyopathy was first mooted by Lundbaek who found myocardial dysfunction in as many as two-thirds of elderly DM patients. ${ }^{1}$ Further expanding on this, Rubier et al in 1972 defined diabetic cardiomyopathy as a 'myocardial dysfunction in patients with diabetes in the absence of coronary artery disease, left ventricular hypertrophy or valvular disease.'2 While all older trials included myocardial infarction as an endpoint, heart failure was either not reported (FIELDS, CARDS, PROACTIVE) or used as an exclusion criteria (UKPDS, DIBHYCAR, DCCT). Heart failure as an endpoint came into the limelight over the last decade when the PDA made cardiovascular safety an integral component of any new antidiabetic drug. Shah et al showed that heart failure after peripheral artery disease is the second most common cardiovascular presentation in diabetes ahead of angina and myocardial infarction. ${ }^{3}$

Financial or Other, Competing Interest: None.

Submission 06-03-2017, Peer Review 14-04-2017,

Acceptance 20-04-2017, Published 27-04-2017.

Corresponding Author:

Dr. Trinath Kumar Mishra,

Bhabha Nagar 2nd Stage,

Berhampur-760004, Orissa.

E-mail:drtkmishra@yahoo.com

DOI: $10.14260 /$ jemds $/ 2017 / 613$

\section{(c) $($ ) $\odot$}

\section{DISCUSSION}

The prevalence of heart failure in the general population is 1 -496 , while it is $12 \%$ in the diabetic population which rises to $22 \%$ in patients $>64$ years (which is the most rapidly growing population segment in western countries). Data from the Framingham Heart Study showed that the prevalence of $\mathrm{HF}$ in diabetics is twice as high in men and five times as high in women. Hamby et al noted that the incidence of diabetes was $22 \%$ in a cohort of diabetic cardiomyopathy as compared to $11 \%$ in the placebo group. An increase in $\mathrm{HbA1c}$ is associated with a higher risk of heart failure incidence (2.3 11.9 per 1000 patient-years over 10 years of follow-up) During a mean follow-up of 2.2 years, each $1 \%$ increase in $\mathrm{HbA1c}$ was associated with an $8 \%$ increase in $\mathrm{HF}$ and for a mean follow-up of 7 years, $\mathrm{HF}$ development increased progressively from $<5 \%$ with an HbA1c of 7.0 to $72 \%$ with an $\mathrm{HbA} 1 \mathrm{c}$ greater than $10 \%$. The risk of $\mathrm{HF}$ in diabetics reduces by $16 \%$ for every $1 \%$ reduction in HbA1c. 4 The average age of developing heart failure is 5.5 years less in diabetics as compared to non-diabetics. Importantly, the risk of HF increases with the age and duration of diabetes. Even in type 1 diabetics, an increase of $1 \%$ in $\mathrm{HbA} 1 \mathrm{c}$ was associated with a $30 \%$ higher risk of HF. Evidence linking HF and DM also comes from the HF trials (SOLVD, ATLAS and V-HeFT II), in which diabetes was found in $19 \%-26 \%$ of patients as compared to $4 \%-6 \%$ in the general population. The presence of diabetes worsens the prognosis, both morbidity and mortality, in heart failure again independently of complicated CAD; $50 \%$ of all diabetics hospitalised for HF die within 3 years. ${ }^{5}$ Diabetes doubles the mortality in HF and triples the risk of SCO in HF and this increased risk is observed in both ischaemic and non-ischaemic HF. Multiple trials such as SAVE, VALIANT, CHARM have unequivocally demonstrated that the risk of hospitalisation for HF is markedly higher in diabetics. A large study including 45,227 patients of $\mathrm{HF}$ (43.6\% of whom were diabetics) found that the 4-year HR of cardiovascular death, non-fatal MI or non-fatal stroke in the diabetic population $(16.5 \%)$ compared to the non-diabetic (13.1\%) was 1,27, $\mathrm{P}<0.001$. While $\mathrm{CV}$ death and overall death was significantly higher in diabetics, diabetics had a $33 \%$ higher risk of hospitalisation for HF. Apart from prognosis, $\mathrm{DM}$ is also implicated in the causation of HF. The major putative mechanisms are chronic under perfusion of liver and 
pancreas, catecholamine excess due to chronic sympathetic overactivity, reduced physical activity and the metabolic effects of $\mathrm{P}$ blockers and diuretics.

A study on HF in diabetics $>60$ years in Netherlands has revealed that $83 \%$ had HFpEF, while $17 \%$ had HFrEF. ${ }^{\circ} \mathrm{A}$ wealth of accumulating data detailing cardiac hypertrophy and diastolic dysfunction has confirmed the presence of diabetic cardiomyopathy. Eguchi et al displayed the relationship between type $2 \mathrm{DM}$ and increased LV mass independent of confounding factors. The Framingham study and Framingham Offspring study both echocardiographically revealed increased $\mathrm{LV}$ wall thickness, end diastolic dimension and LV mass in diabetic females. Echocardiographic parameters of diastolic dysfunction including $\mathrm{E}$ velocity and $\mathrm{E} / \mathrm{E}$ are not only more common in diabetics as compared to non-diabetics, but the severity of diastolic dysfunction also has been demonstrated in the Strong Heart Study to have a linear relationship with HbA1c level. ${ }^{7}$ Comprehensive echocardiographic and Doppler studies have found diastolic dysfunction to be present in up to $60 \%$ of type 1 and type 2 DM patients without CAD. The evidence base for systolic dysfunction is much less robust. While strain imaging studies have detected impairment in longitudinal and radial strain in diabetics, impaired coronary flow reserve as a consequence of endothelial dysfunction, reduced capillary density and interstitial fibrosis may result in ischaemia and consequently systolic dysfunction.

The pathophysiology of diabetic cardiomyopathy is best explained by dividing it into two phenotypes- restrictive (or HFpEF) and dilated (or HFrEF). In the former, the LV cavity is small and the LV walls are thickened due to cardiomyocyte hypertrophy and reactive fibrosis. In the dilated phenotype, the LV cavity is dilated due to cardiomyocyte cell death and large areas of replacement fibrosis. The contributory mechanisms are mainly hyperglycaemia, lipotoxicity and insulin resistance in HFpEF and autoimmunity in HFrEF. Deposition of AGEs and microvascular rarefaction are operative in both the categories (Fig. 1 and 2). ${ }^{8}$

Hyperglycaemia induces production of superoxide by mitochondrial fission leading to impaired activity of NO synthase and reduced production of cGMP. This reduced PKG activity in adjacent cardiomyocytes leading to hypophosphorylation of the contractile protein, titin. ${ }^{9}$ This leads to impaired relaxation of the sarcomere and consequently HFnEF. Hyperglycaemia also increases PKC activity in fibroblasts and augments collagen production. Due to impaired glucose uptake by cardiomyocytes, free fatty acids are preferentially taken up and utilised as a source of energy. This entails a higher oxygen consumption as well as generation of toxic lipid intermediates which impair endothelial function of coronary microvasculature, both of which cause diastolic heart failure. Increased reactive fibrosis seen in the HFpEF phenotype may also be caused by multiple other mechanisms including RAAS that activates TGF01 pathway, AGE/RAGE axis that suppresses collagen turnover by crosslinking, hyperinsulinaemia that impairs fibroblast progenitor cell differentiation and impaired extracellular matrix degradation. ${ }^{10}$

Direct cardiomyocyte death due to lipotoxicity can lead to HFrEF. The proinflammatory cytokines released due to obesity reduce $\mathrm{NO}$ availability in the endothelial cells leading to diastolic dysfunction as described above. Moreover, insulin resistance leads to less efficient energy production in cardiomyocytes due to increased production of uncoupling proteins. Insulin is involved in multiple signalling pathways e.g. PI3k/Akt and their derangement can lead to disproportionate cardiomyocyte hypertrophy. Autoimmunity is more commonly implicated in type $1 \mathrm{DM}$ patients in whom antimyosin antibodies have been isolated and also continuous relapse of troponins from cardiomyocytes has been demonstrated.

Microvascular rarefaction implies a reduced capillary surface area relative to cardiomyocyte surface area. While initially it leads to diastolic dysfunction by reduced NO availability to cardiomyocytes, it may eventually lead to the dilated phenotype by directly causing cell ischaemia and death. AGE deposition in endothelial cells and smooth muscle cells of the microvasculature reduces $\mathrm{NO}$ availability to cardiomyocytes. On the other hand, deposition of AGEs in the interstitium leads to increased free oxygen radical production and cardiomyocyte cell death. Abnormalities in platelet function due to hyperglycaemia and insulin resistance leading to microvascular thrombosis may be another contributory mechanism.

The clinical presentation of the restrictive phenotype of DMCMP may be confused with many other cardiac and noncardiac conditions. The patient complains of shortness of breath and exercise intolerance. Patient is usually obese and on examination has findings of heart failure including pedal oedema, S4, cardiomegaly, rales, raised JVP and other features of right and left ventricular dysfunction. Exclusion of coronary artery disease, congenital, valvular or hypertensive heart disease by angiography and echocardiography and infiltrative heart disease by endomyocardial biopsy (if required) leads then to further echocardiographic evaluation for diastolic dysfunction in the presence of normal LV systolic function. Normal LV systolic function is evidenced by LVEF > $50 \%$ with an LVED volume index $<90 \mathrm{~mL} / \mathrm{m}^{2}$. Doppler criteria for diastolic dysfunction is an E/E' $>15$ or E/E' $8-15$ with added Doppler data from pulmonary venous inflow, LA dilation, atrial fibrillation or raised natriuretic peptides (Fig. 3).

Treatment of HF is the same in both diabetics and nondiabetics. ACE inhibitors, as a class effect, reduce total mortality by $23 \%$ and the combined endpoint of mortality and hospitalisation by 35\% with the greatest benefit seen in the first few months and in the patients with the lowest EF. Among the ARBs, valsartan does not reduce mortality, but reduce the combined endpoint of mortality and hospitalisation for $\mathrm{HF}$ mainly driven by reduced hospitalisation. However, candesartan significantly reduces both mortality and hospitalisation and hence, ACEI may be used in patients who develop ACEI induced cough. Specific data regarding the effects of ACEI or ARB in diabetics versus non-diabetics is not available. Among B blockers, metoprolol succinate, bisoprolol and carvedilol reduce mortality and hospitalisation for heart failure in heart failure patients. Mineralocorticoid receptor antagonists, spironolactone and eplerenone, reduced both total and CV mortality and are considered standard of care in patients with LVEF $<35 \%$ who are already on an ACEI/ARB and 3 blocker. ${ }^{11}$

Use of ARNI has recently been shown to reduce both mortality and hospitalisation in the PARADIGM HF trial. A 
recent analysis of the PARADIGM HF trial showed that patients with a baseline diagnosis of diabetes (35\%) had a higher risk of death or HF hospitalisation. ${ }^{12}$ During the study, an additional $13 \%$ were found to be diabetic (HbA1c > 6.5\%) and $25 \%$ had pre-diabetes (HbA1c $6-6.5 \%$ ). Compared to non-diabetics, pre-diabetics, newly diagnosed diabetics and known diabetics had all significantly and progressively increased incidence of the primary endpoint. Also some benefit of sacubitril-valsartan as compared to enalapril was seen in all the subgroups. Use of ivabradine in HF patients has been shown to reduce 2-year composite outcome of $\mathrm{CV}$ death or hospitalisation. A diabetic sub-study of SHIFT found the prevalence of DM to be $30 \%$, out of whom $32 \%$ were on insulin therapy. The diabetic population had an increase in the primary outcome compared to non-diabetics (HR 1.18). Ivabridine was equally beneficial in both the groups. ${ }^{13}$ Glucose lowering treatment options for heart failure diabetics are limited. The observational studies and the clinical intervention based trials show somewhat conflicting evidence regarding glycaemic control and outcome. A population based study of 16,524 patients in Canada revealed an increased risk of all cause death or HF hospitalisation in patients with a presenting blood sugar $>11.1 \mathrm{mmol} / \mathrm{L}$. On the other hand, the ASTRONAUT trial found no difference in inhospital outcomes between diabetics and non-diabetics. However, post discharge followup showed increased mortality and repeat hospitalisation at around 1 year in ASTRONAUT and EVEREST trial. ${ }^{14}$ Moreover, patients receiving insulin had worse prognosis compared to those on oral drugs or diet restriction. Since 2008, the FDA has mandated the demonstration of cardiovascular safety of all new anti-diabetic drugs. The data on insulin is as of now insufficient and requires further research. An observational study of 554 patients of advanced systolic HF found insulin treated DM to be an independent predictor of mortality. On the contrary, the ORIGIN trial following 12537 patients over 6.2 years found a neutral effect of insulin glargine on development of HF. ${ }^{15}$ In spite of earlier concerns regarding lactic acidosis, a review of 9 cohort studies found metformin to be safe in heart failure and hence recommended except in acute decompensated heart failure or those with other risk factors for lactic acidosis. The sulfonylureas are strongly recommended as second line agents due to their low cost and high efficacy and amongst them the newer drugs including glimepiride and gliclazide may have better cardiovascular safety. TZD use has consistently been associated with increased risk of heart failure as shown in a meta-analysis of 29 placebo-controlled trials (5.3 vs $3.7 \%$ OR 1.59 , 95\% CI $1.34-1.89, \mathrm{p}<0.00001)$. Hence, both the thiazolidinediones (pioglitazone and rosiglitazone) are contraindicated in heart failure. Analysis of the cardiovascular safety of the DPP 4 inhibitor saxagliptin in the SAVOR TIMI 53 trial found early heart failure hospitalisation to be more common in the saxagliptin arm. ${ }^{16}$ While multiple studies with linagliptin and sitagliptin have found no such detrimental effects, the FDA recommends discontinuation of saxagliptin and alogliptin in patients of heart failure. The evaluation of the GLP1 agonist liraglutide in the LEADER trial found it to improve cardiovascular outcomes as compared to placebo. ${ }^{17}$ However, more long-term studies are needed to draw any firm conclusion. The EMPAREG OUTCOME trial revealed that the use of the SGLT2 inhibitor empagliflozin in addition to standard of care reduced the composite endpoint of heart failure hospitalisation and cardiovascular death (by 34\%) as well as the individual components in diabetics with established cardiovascular disease. This may be related to its effects in causing weight loss, reducing plasma volume, lowering systolic blood pressure and/or creating a more ketogenic metabolism. Although, long-term clinical experience is awaited, SGLT2 inhibitors are currently the second line agent of choice in diabetics with cardiovascular disease after metformin.

Commensurate with the approach in research and clinical trials, clinical practice has also focused the management of diabetes on glycaemic control, blood pressure and lipid management and prevention of microvascular complications namely albuminuria, retinopathy and neuropathy. Annual screening for heart failure in diabetics by symptoms, clinical examination and BMP or echocardiography appears to be cost effective and will become more justifiable if evidence based therapy for HFpEF were to be available in the future. The Strong Heart Study showed that the degree of diastolic dysfunction is proportionate to albuminuria even after adjustment for confounders. Hence, it might be worthwhile to perform echo screening in diabetics with albuminuria. The sustained thrust on CAD and the use of mortality reducing therapy including antiplatelets, ACE inhibitors and statins has substantially improved the outcome in this syndrome in both diabetics and non-diabetics. However, the response to treatment for heart failure with guideline directed therapy in diabetics is less ameliorating with less beneficial LV remodelling and larger diuretic requirement at one year of treatment. 18 The outlook for HF, especially HFpEF is likely to improve by better phenotyping of our patients using cluster analyses and bioinformatics.

\section{Clinical DMCMP with Restrictive/HFPEF Phenotype}

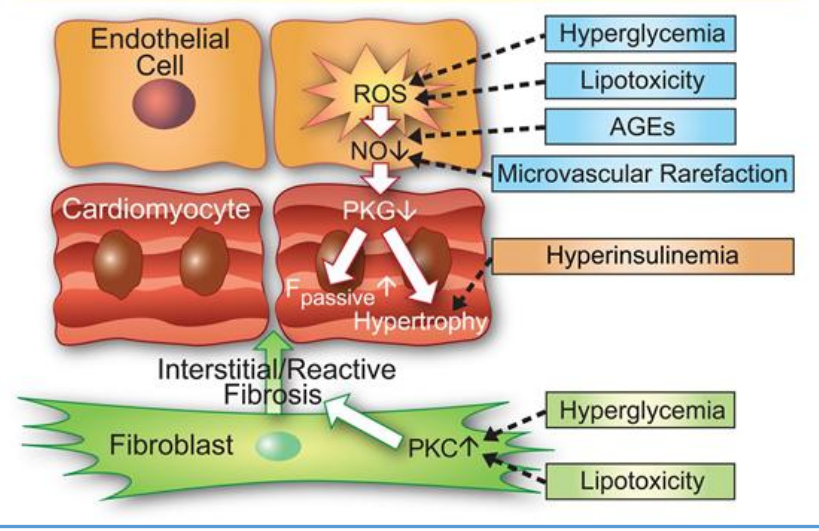

Figure 1. DM-Related Pathophysiological Mechanisms in Diabetes Mellitus-Related Cardiomyopathy with Restrictive/ Heart Failure with Preserved Ejection Fraction Phenotype

From Petar M. Seferovic, Walter J. Paulus; Clinical diabetic cardiomyopathy: a two-faced disease with restrictive and dilated phenotypes. Eur Heart J2015; 36 (27): 1718-1727. 


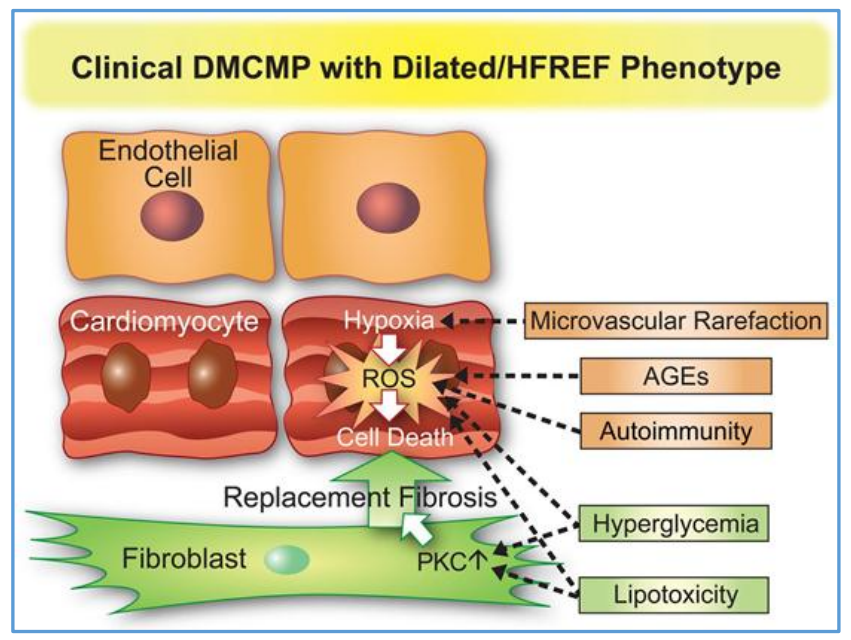

Figure 2. DM-Related Pathophysiological Mechanisms in Diabetes Mellitus-Related Cardiomyopathy with Dilated/ Heart Failure with Reduced Ejection Fraction Phenotype

From Petar M. Seferovic, Walter J. Paulus; Clinical diabetic cardiomyopathy: a two-faced disease with restrictive and dilated phenotypes. Eur Heart J2015; 36 (27): 1718-1727.

DIAGNOSTIC CRITERIA FOR DIABETIC CARDIOMYOPATHY (DMCMP)

\begin{tabular}{|c|c|c|c|}
\hline \multicolumn{4}{|c|}{ DIAGNOSTIC CRITERIA FOR DIABETIC CARDIOMYOPATHY (DMCMP) } \\
\hline & $\begin{array}{c}\text { DMCMP } \\
\text { Restrictive/HFPEF Phenotype }\end{array}$ & & $\begin{array}{c}\text { DMCMP } \\
\text { Dilated/HFREF Phenotype }\end{array}$ \\
\hline 1) & Presence of DM & 1) & Presence of DM \\
\hline 2) & $\begin{array}{l}\text { Exclusion of CAD, valvular or } \\
\text { congenital heart disease }\end{array}$ & 2) & $\begin{array}{l}\text { Exclusion of CAD, valvular or } \\
\text { congenital heart disease }\end{array}$ \\
\hline 3) & $\begin{array}{l}\text { Exclusion of hypertensive heart } \\
\text { disease (=DBP<90mmHg) }\end{array}$ & 3) & $\begin{array}{l}\text { Exclusion of hypertensive heart } \\
\text { disease }(=D B P<90 \mathrm{mmHg})\end{array}$ \\
\hline 4) & $\begin{array}{l}\text { Exclusion of infiltrative heart } \\
\text { disease by endomyocardial biopsy }\end{array}$ & 4) & $\begin{array}{l}\text { Exclusion of viral myocarditis by } \\
\text { endomyocardial biopsy }\end{array}$ \\
\hline 5) & LVEF $>50 \% ;$ LVEDVI $<97 \mathrm{ml} / \mathrm{m}^{2}$ & 5) & LVEF $<50 \% ;$ LVEDVI $>97 \mathrm{ml} / \mathrm{m}^{2}$ \\
\hline 6) & $\begin{array}{l}E / E^{\prime}>15 \text { or } \\
8<E / E^{\prime}<15+L A V I>40 \mathrm{ml} / \mathrm{m}^{2} \text { or } \\
8<E / E^{\prime}<15+B N P>200 \mathrm{pg} / \mathrm{ml} \text { or } \\
8<E / E^{\prime}<15+\text { atrial fibrillation or } \\
8<E / E^{\prime}<15+L V H\left(L V M I+>122 \mathrm{~g} / \mathrm{m}^{2} ;\right. \\
\left.\text { LVMI }^{+}>149 \mathrm{~g} / \mathrm{m}^{2}\right)\end{array}$ & & \\
\hline
\end{tabular}

Figure 3. Diagnostic Criteria for Diabetic Cardiomyopathy

From Petar M. Seferovic, Walter J. Paulus; Clinical diabetic cardiomyopathy: a two-faced disease with restrictive and dilated phenotypes. Eur Heart J2015; 36 (27): 1718-1727.

\section{REFERENCES}

[1] Lundbaek K. Diabetic angiopathy: a specific vascular disease. Lancet 1954;266(6808):377-9.

[2] Rubler S, Dlugash J, Yuceoglu YZ, et al. New type of cardiomyopathy associated with diabetic glomerulosclerosis. Am J Cardiol 1972;30(6):595-602.

[3] Shah AD, Langenberg C, Rapsomaniki E, et al. Type 2 diabetes and incidence of cardiovascular diseases: a cohort study in 1.9 million people. Lancet Diabetes Endocrinol 2015;3(2):105-13.

[4] Stratton IM, Adler Al, Neil HA, et al. Association of glycaemia with macrovascular and microvascular complications of type-2 diabetes (UKPDS 35): prospective observational study. BMJ 2000:321(7258):405-12.
[5] Marx N. Heart failure and diabetes - underestimated, underdiagnosed and poorly understood: a call for action. Diabetes and Vascular Disease Research 2017;14(2):67-8.

[6] Boonman-de Winter LJ, Rutten FH, Cramer MJ, et al. High prevalence of previously unknown heart failure and left ventricuiar dysfunction in patients with type 2 diabetes. Diabetologia 2012;55(8):2154-62.

[7] Hollscher ME, Bode C, Bugger H. Diabetic cardiomyopathy: does the type of diabetes matter? International Journal of Molecular Sciences 2016;17(12):2136.

[8] Seferovic PM, Paulus WJ. Clinical diabetic cardiomyopathy: a two-faced disease with restrictive and dilated phenotypes. Eur Heart J 2015;36(27): 1718-27.

[9] Shenouda SM, Widlansky ME, Chen K, et al. Altered mitochondrial dynamics contributes to endothelial dysfunction in diabetes mellitus. Circulation 2011;124(4):444-53.

[10] Bando YK1, Murohara T. Heart failure as a comorbidity of diabetes: role of dipeptidyl peptidase 4 . J Atheroscler Thromb 2016;23(2):147-54.

[11] Bahtiyar G, Gutterman D, Lebovitz H. Heart failure: a major cardiovascular complication of diabetes mellitus. Curr Diab Rep 2016;16(11):116.

[12] Kristensen SL, Preiss D, Jhund PS, et al. Risk-related to pre-diabetes mellitus and diabetes mellitusin heart failure with reduced ejection fraction: insights from prospective comparison of ARNI with ACEI to determine global mortality and morbidity in heart failure trial. Circ Heart Fail 2016;9(1):e002560.

[13] Bohm M, Robertson M, Ford I, et al. Influence of cardiovascular and noncardiovascular co-morbidities on outcomes and treatment effect of heart rate reduction with ivabradine in stable heart failure (fom the SHIFT trial). Am J Cardiol 2015;116(12):1890-7.

[14] Sarma S, Mentz RJ, Kwasny MJ, et al. Association between diabetes mellitus and post-discharge outcomes in patients hospitalized with heart failure: findings from the EVEREST trial. Eurj Heart Fail 2013;15(2):194-202.

[15] Gerstein HC, Bosch J, Dagenais GR, et al. Basal insulin and cardiovascular and other outcomes in dysglycemia. N Engi J Med 2012;367(4):319-28.

[16] Xu J, Rajaratnam R. Cardiovascular safety of noninsulin pharmacotherapy for type 2 diabetes. Cardiovascular Diabetology 2017;16:18.

[17] Marso SP, Daniels GH, Brown-Frandsen K, et al. Liraglutide and cardiovascular outcomes in type 2 diabetes. N Engl J Med 2016;375(4):311-22.

[18] Kasznicki J, Drzewoski J. Heart failure in the diabetic population - pathophysiology, diagnosis and management. Archives of Medical Science 2014;10(3):546-56. 